\title{
Reconstruction of Hanqing Stadium Scenery Based on Virtual Reality Technology
}

\author{
ShiYu Zhang ${ }^{1}$, YingHao Dong ${ }^{2}$, Tingting Xie ${ }^{1}$, Xinying $\mathrm{Si}^{1}$ and JinZe $\mathrm{Li}^{3}{ }^{*}$ \\ ${ }^{1}$ College of Economics and Management University, Shenyang Aerospace University, Shen Yang, liao Ning, 110135,China \\ 2. College of Information and Computer Engineering, Northeast Forestry University, Harbin, Heilongjiang, 150040, China \\ ${ }^{3}$ College of Mechanical and Electrical Engineering, Shenyang Aerospace University, Shen Yang, liao Ning, 110135,China
}

\begin{abstract}
In order to reproduce the grand look of Hanqing Stadium and restore the most realistic Hanqing Stadium internal layout and structure, a simulation system based on the virtual reality technology for the internal structure layout design of the stadium was designed and developed. Based on the current development trend of virtual reality technology, a three-dimensional dynamic space model was established to restore the real conditions of Hanqing Stadium. By integrating emerging technologies such as panoramic images, panoramic views, and stereo vision into virtual reality technology, the sense of reality was enhanced. 3Dmaxs was used to establish the Hanqing Stadium scene design model, the surrounding and internal scene models were reconstructed, and the experiencers' feelings about the real scene were restored 1:1. The use of technologies such as panoramic images and views enhances the realism and three-dimensionality of the picture.
\end{abstract}

\section{Introduction}

Located on Taishan Road in Huanggu District, Shenyang, Hanqing Stadium was built in 1929 and has a long history. In 2001, Hanqing Stadium was listed as a national key cultural relics protection unit. As the first modern stadium in the country at that time, it could hold more than 30,000 people. Its rostrum is of the Romanesque architectural style, and the horseshoe-shaped stands open to the south, so it is also known as the "Roman-style horseshoe stadium", which fully reflects the design style of the integration of Chinese and Western cultures ${ }^{[1]}$. However, due to the relocation of Shenyang Sport University in 2007, the fate of Hanqing Stadium became rugged. Because it is a cultural relic site and developers cannot develop it, Hanqing Stadium has begun to become dilapidated. The plastic field suffered severe damage, the house near the stand was on fire, and the text on the stone tablet had become blurred. However, as a national key cultural relics protection unit, Hanqing Stadium still has its historical research value.

Using virtual reality technology, through the simulating construction of Hanqing Stadium's style, appearance, structure composition, and spatial details, the historical grand scene of Hanqing Stadium was restored. Scientific and technological means were applied to display the building through visualization technology ${ }^{[2]}$. The application of this technology has made a great contribution to the historical research and educational research value of Hanqing Stadium.

Virtual reality technology is generated using computer graphics systems and various control system equipment. It relies on an interactive environment ${ }^{[3]}$ to present users with visual, auditory, smell, touch and other immersive sensory simulations. The rise and development of virtual reality technology is a leap forward for the development of science and technology in China, and it has contributed a new force to the development of computers. Therefore, it has been applied to many current fields including medical, engineering, military, aviation and navigation, etc. The development of this technology is becoming more and more popular.

The emergence of VR technology ${ }^{[4]}$ has also made important contributions to the development of new products. The advantages of VR technology, such as low cost, high efficiency, and ultra-high transmission speed, have made scholars in many countries notice this technology. China has also increased its research efforts on this technology. With the rapid development of computer science and technology in China, virtual reality technology has been widely used in the realization of venues, architecture, and design concepts. ${ }^{[5]}$ On the basis of informatization design methods, the characteristics and advantages of VR technology are used to analyze the design of Hanqing Stadium, so as to construct realistic scenes, which enables users to form a brand-new visual experience, ${ }^{[6]}$ effectively improves visual effects and quality, ${ }^{[7]}$ thus offering immersive experiences.

The existing papers related to Hanqing Stadium [8] mainly focus on the analysis of the environmental structure, research significance and value of the stadium, ${ }^{[9]}$ and have not systematically proposed research methods and specific technologies. This paper proposes a specific

*Corresponding author's e-mail: 183406040107@email.sau.edu.cn 
technical plan for the design of the stadium's scene reproduction, so as to implement the overall display of the stadium and make up for the shortcomings of current related academic papers.

\section{System design plan}

\subsection{Overview of the system}

The recurring stadium solution is based on the VR somatosensory interactive virtual reality device HTC VIVE. Based on HTC VIVE's somatosensory interaction technology[10], it has the ability to track gesture information to complete operations, and can provide users with a better experience. ${ }^{[11]}$ Virtual reality technology (VR) and environment design provide an experience of immersion, interaction and simulation. The SQL structured query language was used to establish contacts and communicate with multiple databases. In order to visualize the image scene and realize the visual management of the assembly relationship, a threedimensional model was established to truly restore the stadium picture. The addition of panoramic images and panoramic videos made the stadium look stronger. The technology system was finally packaged in desktop-level programs through $\mathrm{C \#}$ for people to use and conduct research with.

\subsection{The key technology of the system}

\subsubsection{HTC VIVE interactive device, Unity3D engine}

To fully display the various venues and sports facilities in the stadium and determine the location of objects, Unity3D was used as the main development platform, and interactive equipment HTC VIVE was used to complete the development of the Hanqing Stadium prototype system. ${ }^{[12]}$ The HTC VIVE head-mounted interactive device consists of a head-mounted display, a laser sensor locator, and a handle controller. The device can display with different effects through the binoculars, so as to present immersive 3D virtual pictures after different scenes are superimposed when the user is watching with binoculars. ${ }^{[13]}$ It combines cloud computing, Internet of Things, big data, and other technologies to realize the intelligent experience and viewing process of Hanqing Stadium, and it takes AI intelligent space navigation, intelligent dispatch control, real-time detection of space environment, emergency handling of emergencies and other technologies as the reference basis for space location.

\subsubsection{Acquisition of 3D data and stadium modeling}

As one of the technologies used in the 3D scene reproduction of the stadium, the acquisition of $3 \mathrm{D}$ data is a key part that restricts the location information. The visual presentation of Hanqing Stadium is an experience process from the overall scene to the partial scene, from the large-scale surface to the small-scale orientation. The establishment of a three-dimensional stadium model is an important part of three-dimensional visualization.

Firstly,3DMAX software was used to build a threedimensional model, and the basic simple geometry was used to construct the complex geometry required for the visual presentation of Hanqing Stadium to meet the realistic visual experience. Secondly,In the use of complex geometry modeling, by using multiple two-dimensional geometry and three-dimensional geometry through a series of superimposition and combination, the threedimensional complex geometry required for the construction of the stadium was obtained. Finally, a threedimensional model was generated from the geometry and the three-dimensional image through Boolean operations. [14] Through the data collection and analysis of various facilities, buildings, runways, and sports equipment in the stadium, three-dimensional models of different areas such as outdoor sports ring, natural ecological ring, national fitness ring, and garden tour ring were established to provide users with an intuitive and visual stadium environment.

\subsubsection{Acquisition of panoramic images and panoramic videos}

To solve the issue of the monotonousness of traditional 3D modeling data, based on panoramic image and panoramic video acquisition technology, ${ }^{[15]}$ a dynamic interactive virtual space was built. For the panoramic image, it was planned to adopt two methods to obtain the panoramic image. The first is the method of collecting multiple sets of pictures to form a panoramic image, using panoramic image splicing technology based on image rendering; the second is to use a professional panoramic camera, set appropriate camera calibration parameters in advance, and automatically obtain panoramic images. For the panoramic video, the $3 \mathrm{D}$ camera was used to obtain the 360-degree all-round shooting of the required field and scenery. Panoramic images and panoramic videos are new types of three-dimensional data acquisition methods, which have the characteristics of full viewing angle, strong sense of reality, and high current status, and can display various topography and landforms in more detail. It has a high resolution and three-dimensional effect combined with virtual reality to enhance the immersive experience for users.

\subsubsection{Demonstration of part of the operating system}

According to the content involved in the realization of virtual reality technology in the stadium, the operating system consists of five parts for demonstration, including demonstration of the external scene of the stadium, demonstration of the outline scene of the stadium, demonstration of the layout of the stadium, demonstration of the internal facilities of the stadium, and dynamic demonstration of the functions of the stadium. 


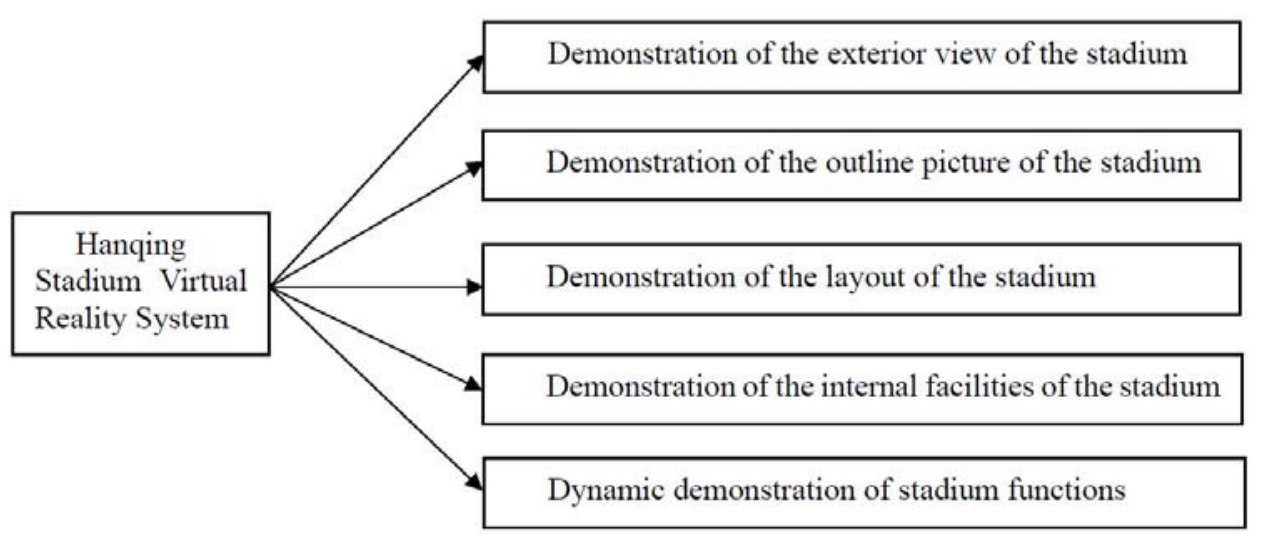

Fig1.Demonstration diagram of Hanqing Stadium virtual reality system

\subsubsection{Model building process analysis [16]}

The overall visualization model of Hanqing Stadium was established by 3DMaxs. 3DMaxs is suitable for a series of modeling productions such as architectural design, 3D animation, and dynamic scenes, which have strong flexibility and are professional tools for making architectural models. 3DMaxs has good guidance nature, maneuverability and practicability in the finished renderings, and has detailed modeling parameters.
Compared with 3DMaxs, Maya is more suitable for model establishment of film and television scenes and animation environments, and 3DMaxs has high work efficiency and strong operability. On this basis, for Hanqing Stadium's in-field model design, 3DMaxs modeling software was used, and PixyzStudio was imported to specifically subdivide different venues or facilities in the stadium, and the model was revised, optimized, and exported. After further processing the Hanqing Stadium model with Cinema4D, it was imported into Unity for use, which effectively realized the lightweight conversion and format conversion of the model data.

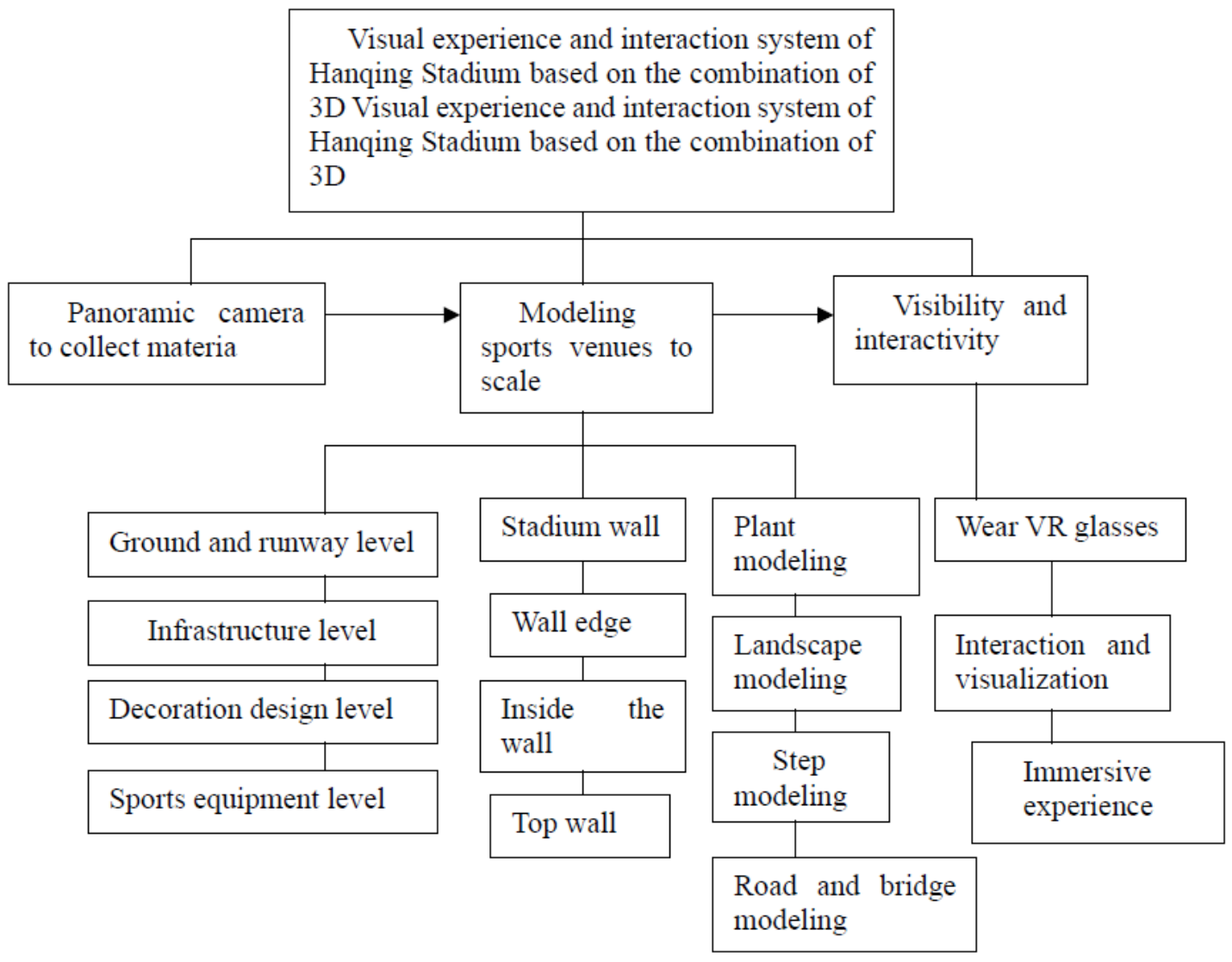

Fig2.Hanqing Stadium interactive experience system block diagram 


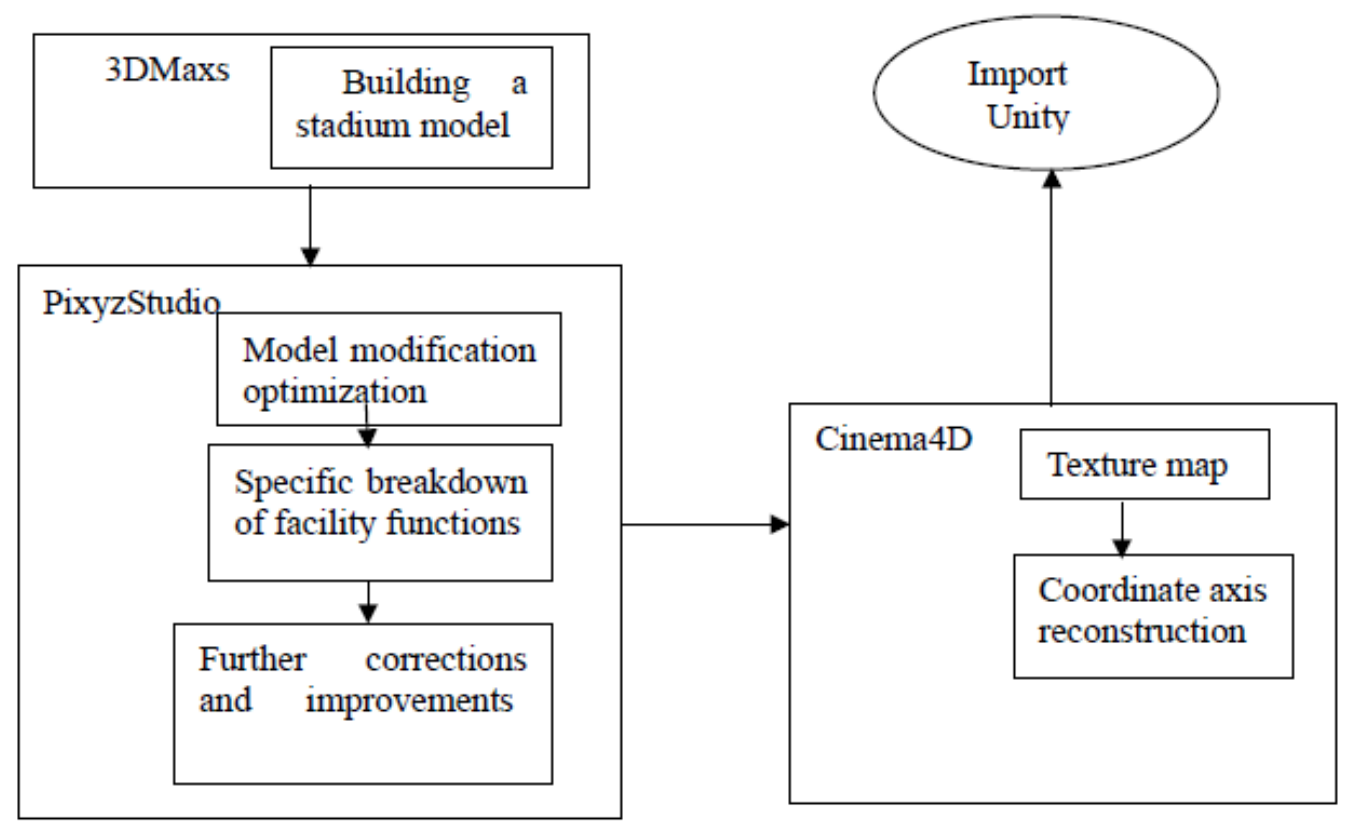

Fig3.Hanqing Stadium model export process diagram

\section{Running results [17]}

The abandoned Hanqing Stadium still has values in many senses. For example, the overall design of Hanqing Stadium perfectly embodies the integration of the central culture. It covers a large area and is a national key cultural relics protection unit, which makes the reconstruction of Hanqing Stadium possible. However, Hanqing Stadium is still in an abandoned state. It is difficult for the public to experience the grandeur of the stadium. Therefore, it is feasible to reshape the whole picture of Hanqing Stadium through virtual reality, which is an effective measure for the public to experience the stadium. Using VR virtual reality simulation, the appearance of the stadium was reproduced, and the large stadium of more than 3,000 square meters was reproduced, so that users can experience and feel the stadium. Light perception was applied to achieve such a user's experience.

As the system is running, the user enters the simulated real world, enters the main page, and sees the view outside Hanqing Stadium. In virtual reality, on the left-handed handle, the user can see the floor plan of Hanqing Stadium. During the experience, the user holds the two handles with
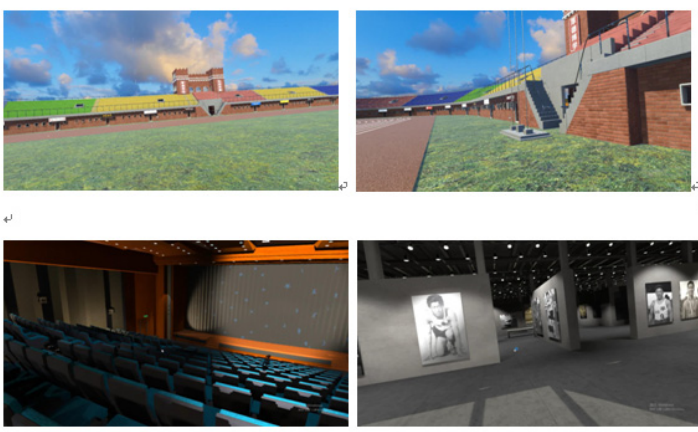

both hands, puts the handle on the text on the floor plan, and releases it to the scene corresponding to the text. In the scene, the user can manipulate the handle to walk and watch the simulated real world in $360^{\circ}$.

During the simulation of Hanqing Stadium in virtual reality, with multiple realistic designs such as sound effects and light perception, Hanqing Stadium can be reproduced truthfully. Sound effects and light perception can simulate the real effects of theaters, bars, and circular racetracks in real stadiums. In order to enhance the experience, the control of the light perception was made, and the simulation of the cloudy sky was strengthened, so as to bring the user into the stadium. The aperture and animation were strengthened, so that when users walk in the stadium, there are apertures and arcs. The interactive design of HTC Vive interactive device was used to fully demonstrate the real effect of simulation. During of scene transition, the connection between the animation effect and the scene is strengthened to reflect the authenticity of the scene and make the experience smoother. All of the measures are to enhance the details, strictly control each part, improve the environment atmosphere, emphasize comfort, interactivity, and authenticity, and enhance the sense of immersion.

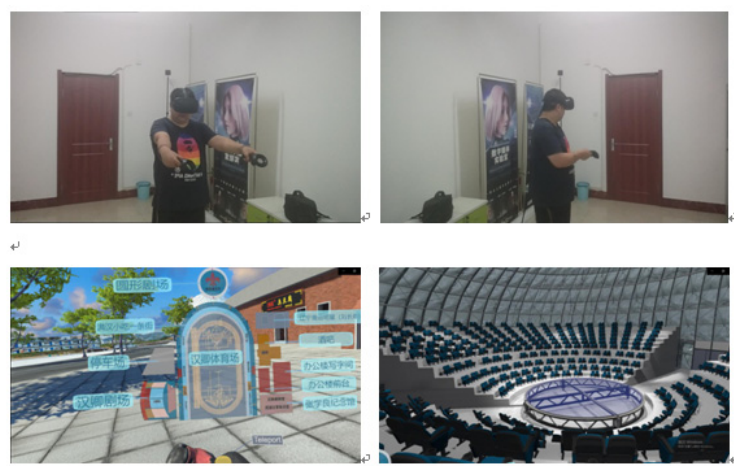



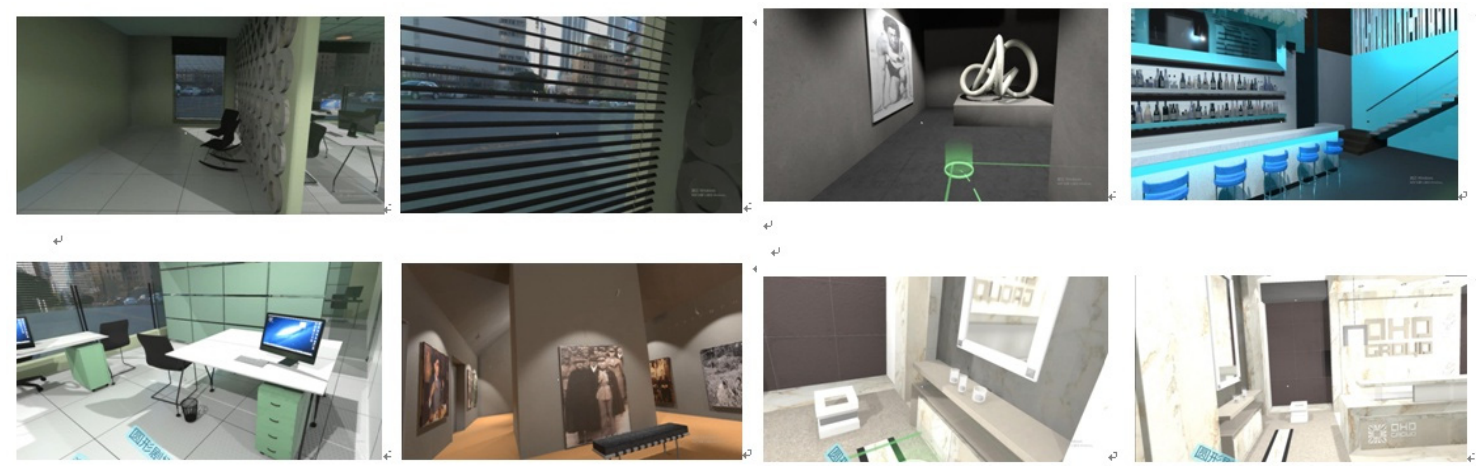

Fig4. Part of the scene

\section{Conclusion}

Based on virtual reality technology (VR) this system reproduces all-round scenes of Hanqing Stadium's internal venues and facilities. Based on the development of the existing $3 \mathrm{D}$ visualization technology, this study has realized the human-computer interactive upgrade, which brings the users natural and comfortable experiences, and enhances the sense of reality and realism in the projection. The acquisition technology of panoramic images and panoramic videos solves the issue of the monotonousness of traditional 3D modeling, and meticulously depicts the different topography of Hanqing Stadium, giving users an immersive experience, letting the users know about the truthful look of Hanqing Stadium without leaving home.

Based on the data analysis obtained from the experiment, the use of virtual reality technology (VR) to analyze the structure of Hanqing Stadium has the following research value. (1) Experiencers can cross geographical restrictions and have a comprehensive experience of the stadium's internal environment without leaving their homes. (2) This system technology can be used to pre-design the renderings of the relevant building products, which is convenient, true and fast. (3) In reality, it can avoid damage to the stadium facilities when the experiencer visits the stadium. (4) The sports culture and related sports knowledge of the stadium can be popularized through the simulated physical objects, models, various experience facilities and demonstration facilities of the stadium. (5) Breaking the visit limitation that may occur when traditional users visit the building and the limitation of incomplete information from the narration of human staff, this system can present the full picture of the stadium in all directions and multiple levels for detailed introduction. (6) The autonomy of the user is fully manifested, and the user can experience, watch and learn at any time and area suitable by actual needs. (7) The system integrates the fun and interactivity in the experience process, and brings the experience with visual shock and surprise.

\section{Reference cited}

1. Sui Hao, Chen Guanru. Research on the Overall Display System of Fengtian Hanqing Stadium [J]. Popular Art,2018(22):104-105.

2. Han Zhiren, Liu Xiaonong, Wu Meng, Liu Baoming.
Research on 3D Visualization Technology of Aircraft Component Assembly Schedule Based on CATIA Composer $[\mathrm{J} / \mathrm{OL}]$. Aviation manufacturing technology: $\quad 1-9 \quad$ [2021-05-10]. http://kns.cnki.net/kcms/detail/11.4387.V.20210510. 0900.004.html.

3. Wang Peng. Contextual framework based on interactive machine translation environment [J]. Journal of University of Science and Technology Beijing (Social Science Edition), 201,37(02):138-146.

4. Liu Ping. Application of Virtual Reality Technology in 3D Product Detail Display [J]. Modern Electronic Technology, 201,44(09):162-165.

5. Yu Zhiqiang. Modern Building Environment Design Supported by Virtual Reality Technology [J]. Sichuan Cement,2020(09):324-325.

6. $\mathrm{Hu}$ Xiaoling, Tan Yongping. Application of virtual reality technology in architectural decoration design practice teaching $[\mathrm{J}]$. Guangxi Education,2020(43):187-189+192.

7. Shen F X. Research on image compression sensing reconstruction algorithm based on visual communication effect $[\mathrm{J}]$. Microcomputer Applications, 201,37(04):61-65.

8. Gu Aihua, Tong Xi, Zhou Huan. Study on the Realization Path of Material Cultural Heritage Value in Shenyang -- Discussion on the Feasibility of Establishing Northeastern University Site Park [A]. Shenyang Municipal Committee of the Communist Party of China, Shenyang Municipal People's Government, International Academy of Production Engineering, China Mechanical Engineering Society. Shenyang Municipal Committee of the Communist Party of China, Shenyang Municipal People's Government, International Production Engineering Academy, Chinese Mechanical Engineering Society: Shenyang Association of Science and Technology,2019:4. Design and Implementation of 3D Virtual Tour System Based on Panoramic Technology $\quad[\mathrm{J}]$. Technology and Market,2021,28(03):92-93.

9. Li Yao. Study on the Interaction between Architectural Structure and Interior Space under Parametric Design -- Taking Alien Space Design as an Example [J]. Tomorrow Style,2021(05):118-119.

10. Lei Zhang, Xiaobin Xu, Jia He, Kaiyuan Zhu, Minzhou Luo, Zhiying Tan. Two-dimensional LiDAR 
and Camera Calibration Method Based on Indoor Structure Characteristics [J]. Acta Photonica Sinica,2020,49(12):67-78.

11. Yuan Hao. Virtual Reality Building Teaching Method Based on HTC VIVE [J]. Shanxi Architecture,2017,43(24):256-257.

12. SHU Jun.Implementation of Campus Display and Interactive System Based on Unity3D and HTC Vive [D]. Nanchang Hangkong University,2019.

13. Mi Dan, Wang Qin, Wang Chunjie. Virtual reality roaming of model house based on Unity3D [J]. Modern Computer,2019(24):97-100.

14. LUO Xu. Method Analysis and Technique Discussion on 3DMAX Modeling [J]. Computer Knowledge and Technology,2020,16(35):207-208+213.

15. Zheng Xiaoli. Design and Implementation of 3D Virtual Tour System Based on Panoramic Technology [J]. Technology and Market,2021,28(03):92-93.

16. Ma Leilei. Modeling and Hierarchical Rendering System for Large-scale Scenes [D]. Shandong University,2020.

17. ZHAO Bing. Analysis of VR natural disaster scene experience simulation system $[\mathrm{J}]$. Electronic World,2020(08):23-24. 